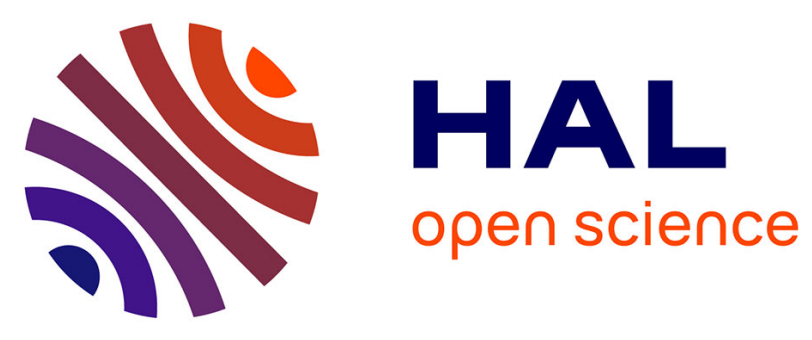

\title{
3D eddy currents computation by BEM using the modified magnetic vector potential and the reduced magnetic scalar potential
}

Quang-Anh Phan, Gérard Meunier, Olivier Chadebec, Jean-michel Guichon, Bertrand Bannwarth

\section{To cite this version:}

Quang-Anh Phan, Gérard Meunier, Olivier Chadebec, Jean-michel Guichon, Bertrand Bannwarth. 3D eddy currents computation by BEM using the modified magnetic vector potential and the reduced magnetic scalar potential. International Journal of Numerical Modelling: Electronic Networks, Devices and Fields, 2020, 10.1002/jnm.2642 . hal-02364312v1

\section{HAL Id: hal-02364312 \\ https://hal.science/hal-02364312v1}

Submitted on 14 Nov 2019 (v1), last revised 9 Nov 2020 (v2)

HAL is a multi-disciplinary open access archive for the deposit and dissemination of scientific research documents, whether they are published or not. The documents may come from teaching and research institutions in France or abroad, or from public or private research centers.
L'archive ouverte pluridisciplinaire HAL, est destinée au dépôt et à la diffusion de documents scientifiques de niveau recherche, publiés ou non, émanant des établissements d'enseignement et de recherche français ou étrangers, des laboratoires publics ou privés. 


\section{D Eddy Currents computation by BEM using the modified magnetic vector potential and the reduced magnetic scalar potential}

\section{Quang-Anh Phan | Gerard Meunier | Olivier Chadebec | Jean-Michel Guichon | Bertrand Bannwarth}

Univ. Grenoble Alpes, CNRS, Grenoble INP*, G2Elab, 38000 Grenoble, France.

* Institute of Engineering Univ. Grenoble Alpes

Correspondence

Email: quang-anh.phan@g2elab.grenoble-inp.fr

Present Address

G2Elab, 21 avenue des Martyrs, 38031

Grenoble, FRANCE

\begin{abstract}
Summary
A new Boundary Element Method (BEM) formulation has been developed for the computation of $3 \mathrm{D}$ eddy currents in both magnetic and conductive regions. The modified magnetic vector potential $\mathbf{A}^{*}$ in the active regions and the reduced magnetic scalar potential $\varphi$ in the air are used as unknown variables. The formulation is limited to low-frequency magneto-harmonic problems associated to linear, homogeneous, isotropic and simply-connected regions. In such a configuration, it leads to accurate results with a reduced number of degrees of freedom located on a surface mesh delimiting the air/material interface.
\end{abstract}

KEYWORDS:

Boundary element method, 3D eddy current, quasi-statics, modified magnetic vector potential, reduced magnetic scalar potential

\section{1 | INTRODUCTION}

At low frequencies, the quasi-static approximation enables the simplification of Maxwell equations by neglecting the current displacement term. In this context, the Boundary Element Method (BEM) has been used for a long time to solve 3D eddy currents problems.The advantage of this method is obvious since it requires only the discretization of the surface between the conductive material and the air region. With BEM, the number of elements of the mesh is much smaller compared to the FE (Finite Elements)-like methods and the dimension of the problem is strongly reduced. The counterpart is its limitation to linear, homogeneous and isotropic materials and the getting of fully dense matrix systems. Since the beginning of the 90's, many formulations has been developed based on different magnetic and/or electric quantities as degrees of freedom. Rucker 3 , Tsuboi ${ }^{4}$ developed formulations based on the magnetic vector potential and the electric scalar potential. Mayergoyz ${ }^{6}$, Kalaichelvan ${ }^{7}$, Harrington ${ }^{8}$ solved the integral equations with equivalent electric current and magnetic charge as unknowns. Stratton ${ }^{16}$, Zheng ${ }^{12}$, Hiptmair ${ }^{10}$ have developed formulations based on the equivalent electric and magnetic surface currents. In this paper, we present a new BEM formulation with the modified magnetic vector potential $\mathbf{A}^{*}$ and the reduced magnetic scalar potential $\varphi$ as unknowns. Let us notice that this formulation can only handle problems containing simply connected conductive domains.

\section{2 | EDDY CURRENT PROBLEM}

Let us consider a both magnetic and conductive region $\Omega_{1}$ delimited by the surface $\Gamma_{1}$ with a permeability $\mu_{1}$ and a conductivity $\sigma_{1}$. The exciting magnetic field $\mathbf{H}_{\mathrm{s}}$ is created by an external current $\mathbf{J}_{\mathrm{s}}$ flowing in a coil which is located in the free air region $\Omega_{0}$ as shown in FIGURE 1 Thanks to the quasi-static assumption where displacement currents are neglected, we can write the equations of the magnetic and electric fields in the conductor 


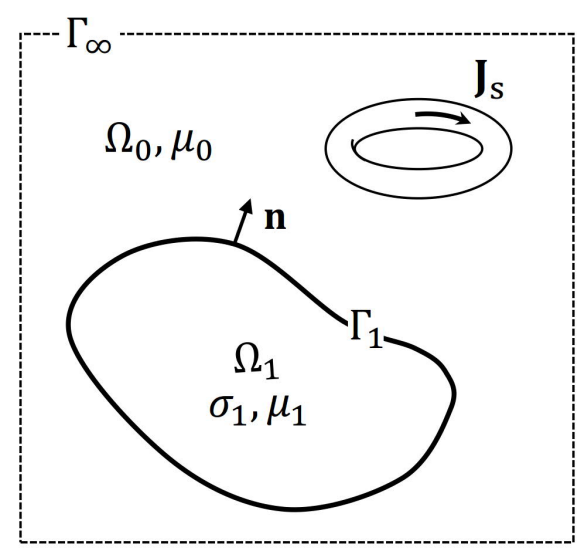

FIGURE 1 Eddy current problem.

as follows:

$$
\left\{\begin{array}{l}
\nabla \times \mathbf{H}=\sigma_{1} \mathbf{E} \\
\nabla \times \mathbf{E}=-\mathbf{j} \omega \mathbf{B}
\end{array}\right.
$$

The modified magnetic vector potential $\mathbf{A}^{*}$ in the material is defined by the relations:

$$
\left\{\begin{array}{l}
\mathbf{E}=-\mathrm{j} \omega \mathbf{A}-\nabla \mathbf{V}=-\mathrm{j} \omega \mathbf{A}^{*} \\
\nabla \times \mathbf{A}^{*}=\mathbf{B}
\end{array}\right.
$$

From 1 and 2 , the potential $\mathbf{A}^{*}$ satisfies the Helmholtz equation:

$$
\nabla \times \nabla \times \mathbf{A}^{*}+j \omega \sigma_{1} \mu_{1} \mathbf{A}^{*}=0
$$

The uniqueness of vector potential is ensured by the gauge $\nabla \cdot \mathbf{A}^{*}=0$. Outside the conductor, the magnetic field $\mathbf{H}$ is irrotational. Therefore, it can be derived from a reduced magnetic scalar potential $\varphi$ :

$$
\mathbf{H}=\mathbf{H}_{s}-\nabla \varphi
$$

Furthermore, this field is solenoidal in the free region $\Omega_{0}$ i.e $\nabla \cdot \mathbf{H}_{0}=0$, leading to the Laplace equation for $\varphi$ :

$$
\Delta \varphi=0
$$

Our problem is to find the solution of the equations $\left[3\right.$ and $[5]$ which satisfies at the the boundary conditions on $\Gamma_{1}$ :

$$
\left\{\begin{array}{l}
(\mathbf{n} \times \mathbf{H})_{1}=(\mathbf{n} \times \mathbf{H})_{0} \\
\mu_{1}(\mathbf{n} \cdot \mathbf{H})_{1}=\mu_{0}(\mathbf{n} \cdot \mathbf{H})_{0}
\end{array}\right.
$$

where the index 0 or 1 indicates that a quantity belongs to the domain $\Omega_{0}$ or $\Omega_{1}$ respectively. After some algebras, these conditions are changed into relations linking $\mathbf{A}^{*}$ and $\varphi$ on the boundary of $\Omega_{1}$ :

$$
\left\{\begin{array}{l}
\mathbf{n} \times\left(\nabla \times \mathbf{A}^{*}\right)=\mu_{1}\left(\mathbf{n} \times \mathbf{H}_{\mathbf{s}}-\mathbf{n} \times \nabla \varphi\right) \\
\mathbf{n} \cdot\left(\nabla \times \mathbf{A}^{*}\right)=\mu_{0}\left(\mathbf{n} \cdot \mathbf{H}_{\mathbf{s}}-\mathbf{n} \cdot \nabla \varphi\right)
\end{array}\right.
$$

\section{3 | BOUNDARY INTEGRAL EQUATION}

Using the vector Green's identity proposed in 1 , an integral equation for the modified vector potential in the conductive region is obtained :

$$
\int_{\Omega_{1}}\left[\left(\nabla \cdot \mathbf{A}^{*}\right) \nabla G+\mathbf{A}^{*} \Delta G+G\left(\nabla \times \nabla \times \mathbf{A}^{*}\right)\right] d \Omega=\oint_{\Gamma_{1}}\left[\left(\mathbf{n} \cdot \mathbf{A}^{*}\right) \cdot \nabla G+\left(\mathbf{n} \times \mathbf{A}^{*}\right) \times \nabla G+\left(\mathbf{n} \times \nabla \times \mathbf{A}^{*}\right) G\right] d \Gamma
$$

where $\mathbf{n}$ is outward unit normal vector to the boundary $\Gamma_{1}$ of the region $\Omega_{1}$. The Green function $\mathrm{G}$ used in this integral equation is a solution of the equation:

$$
\Delta G_{P}(P, Q)-j \omega \mu_{1} \sigma_{1} G(P, Q)+\delta(P, Q)=0
$$


where $\mathrm{Q}$ is the integration point and $\mathrm{P}$ is the observation point. $\delta$ denotes a Dirac pulse occurring at $\mathrm{P}$. For $3 \mathrm{D}$ problems, the complex kernel $\mathrm{G}$ is expressed by:

$$
G=\frac{1}{4 \pi r} e^{-(1+j) k r}
$$

where $\mathrm{k}=\sqrt{\frac{\omega \mu_{1} \sigma_{1}}{2}}$ and $\mathrm{r}=\mathrm{PQ}$. Replacing $\Delta \mathrm{G}_{1}$ in 8 by 9 and by noticing that $\nabla \cdot \mathbf{A}^{*}=0$ and $\mathbf{n} \cdot \mathbf{A}^{*}=0$, we get the integral boundary equation of modified vector potential as follows:

$$
h_{1} \mathbf{A}^{*}=-\oint_{\Gamma_{1}}\left[\left(\mathbf{n} \times \mathbf{A}^{*}\right) \times \nabla G+\left(\mathbf{n} \times \nabla \times \mathbf{A}^{*}\right) G\right] d \Gamma
$$

where the value of the coefficient $h_{1}$ depends on point position $\mathrm{P}$ where the integral expression is written.

$\mathrm{h}_{1}=0$ if $\mathrm{P}$ is outside $\Omega_{1}$

$\mathrm{h}_{1}=1$ if $\mathrm{P}$ is inside $\Omega_{1}$

$\mathrm{h}_{1}=\frac{\Omega}{4 \pi}$ if $\mathrm{P}$ is on the boundary surface $\Gamma_{1}, \Omega$ being the solid angle spanning $\Gamma_{1}$ from the observation point $\mathrm{P}(\Omega=2 \pi$ if the surface is regular). Applying the scalar Green's theorem to the scalar potential $\varphi$ with the Green's function $\mathrm{G}_{0}=\frac{1}{\mathrm{r}}$, we get:

$$
\int_{\Omega_{0}}\left[\left(\varphi \Delta G_{0}-G_{0} \Delta \varphi\right)\right] d \Omega=\oint_{\Gamma_{1}}\left[\left(\mathbf{n} \cdot \nabla G_{0}\right) \varphi-(\mathbf{n} \cdot \nabla \varphi) G_{0}\right] d \Gamma
$$

where $\mathrm{G}_{0}=\frac{1}{\mathrm{r}}$ is the fundamental solution of the Laplace equation and which satisfies: $\Delta \mathrm{G}_{0 \mathrm{P}}(\mathrm{P}, \mathrm{Q})+\delta(\mathrm{P}, \mathrm{Q})=0$. Applying this relation to the left side of the above equation, we finally get the boundary integral equation of $\varphi$ :

$$
h_{0} \varphi=\oint_{\Gamma_{1}}\left[\left(\mathbf{n} \cdot \nabla G_{0}\right) \varphi-(\mathbf{n} \cdot \nabla \varphi) G_{0}\right] d \Gamma
$$

where

$\mathrm{h}_{0}=0$ if $\mathrm{P}$ is outside $\Omega_{0}$

$\mathrm{h}_{0}=1$ if $\mathrm{P}$ is inside $\Omega_{0}$

$\mathrm{h}_{0}=\frac{\Omega}{4 \pi}$ if $\mathrm{P}$ is on boundary surface $\Gamma_{1}$. The integral boundary equations system is obtained as follows:

$$
\left\{\begin{array}{l}
\mathrm{h}_{1} \mathbf{A}^{*}=-\oint_{\Gamma_{1}}\left[\left(\mathbf{n} \times \mathbf{A}^{*}\right) \times \nabla \mathrm{G}+\left(\mathbf{n} \times \nabla \times \mathbf{A}^{*}\right) \mathrm{G}\right] \mathrm{d} \Gamma \\
\mathrm{h}_{0} \varphi=\oint_{\Gamma_{1}}\left[\left(\mathbf{n} \cdot \nabla \mathrm{G}_{0}\right) \varphi-(\mathbf{n} \cdot \nabla \varphi) \mathrm{G}_{0}\right] \mathrm{d} \Gamma
\end{array}\right.
$$

Both terms $\left(\mathbf{n} \times \nabla \times \mathbf{A}^{*}\right)$ and $(\mathbf{n} . \nabla \varphi)$ can be developed based on the boundary conditions in 7]. Finally, we get the BEM formulation $\mathbf{A}^{*}-\varphi$ :

$$
\left\{\begin{array}{l}
-\mu_{1} \oint_{\Gamma_{1}}\left(\mathbf{n} \times \mathbf{H}_{\mathrm{s}}\right) \mathrm{G}_{1} \mathrm{~d} \Gamma=\mathrm{h}_{1} \mathbf{A}^{*}+\oint_{\Gamma_{1}}\left(\mathbf{n} \times \mathbf{A}^{*}\right) \times \nabla \mathrm{G}_{1} \mathrm{~d} \Gamma-\mu_{1} \oint_{\Gamma_{1}}(\mathbf{n} \times \nabla \varphi) \mathrm{G}_{1} \mathrm{~d} \Gamma \\
-\oint_{\Gamma_{1}}\left(\mathbf{n} \cdot \mathbf{H}_{\mathrm{s}}\right) \mathrm{G}_{0} \mathrm{~d} \Gamma=\mathrm{h}_{0} \varphi-\oint_{\Gamma_{1}}\left(\mathbf{n} \cdot \nabla \mathrm{G}_{0}\right) \varphi \mathrm{d} \Gamma-\frac{1}{\mu_{0}} \oint_{\Gamma_{1}} \mathbf{n} \cdot\left(\nabla \times \mathbf{A}^{*}\right) \mathrm{G}_{0} \mathrm{~d} \Gamma
\end{array}\right.
$$

The resolution of this system of equations leads only to $\mathbf{A}^{*}$ and $\varphi$ on the boundary. Some additional computations has to be done to determine several quantities in the volume domain. Using the equation of $\mathbf{A}^{*}$ in 14 . with the coefficient $\mathrm{h}_{1}=1$, the eddy current density inside the conductor is computed as follows:

$$
\mathbf{J}=j \omega \sigma_{1} \oint_{\Gamma_{1}}\left[\left(\mathbf{n} \times \mathbf{A}^{*}\right) \times \nabla G_{1}+\left(\mathbf{n} \times \nabla \times \mathbf{A}^{*}\right) G_{1}\right] d \Gamma
$$

In order to obtain the total magnetic field in the air, we need to compute the gradient of $\varphi$ in 4]. It is obtained by applying gradient operator to [13:

$$
\nabla \varphi=\oint_{\Gamma_{1}}\left[\nabla\left(\mathbf{n} \cdot \nabla G_{0}\right) \varphi-(\mathbf{n} \cdot \nabla \varphi) \nabla G_{0}\right] d \Gamma
$$

Magnetic field $\mathbf{H}$ in the air can also be calculated with the integral equation boundary written for $\mathbf{H}_{0}$ as proposed in 1112 :

$$
\mathbf{H}_{0}=\mathbf{H}_{s}+\oint_{\Gamma_{1}}\left(\mathbf{n} \times \mathbf{H}_{s}\right) \times \nabla G_{0} d \Gamma+\oint_{\Gamma_{1}} \mathbf{n} \cdot\left(\nabla \times \mathbf{A}^{*}\right) \nabla G_{0} d \Gamma-\oint_{\Gamma_{1}}(\mathbf{n} \times \nabla \varphi) \times \nabla G_{0} d \Gamma
$$

To determine the Joule losses, Poynting theorem can be used since it can be applied directly to the $\Gamma_{1}$ surface. After some algebras, an expression for the losses is get:

$$
P_{\text {loss }}=\frac{1}{2} \operatorname{Re}\left(\oint_{\Gamma_{1}}\left(\mathbf{n} \times \mathbf{H}_{1}\right) \cdot \tilde{\mathbf{E}_{1}} d \Gamma\right)=\operatorname{Re}\left(-\frac{j \omega}{2} \oint_{\Gamma_{1}}\left(\mathbf{n} \times \mathbf{H}_{s}-\mathbf{n} \times \nabla \varphi\right) \cdot \tilde{\mathbf{A}}^{*} d \Gamma\right)
$$

where $\tilde{\mathbf{E}}_{1}$ and $\tilde{\mathbf{A}}^{*}$ denotes the complex conjugate of electric field $\mathbf{E}_{1}$ and $\mathbf{A}^{*}$ respectively. 


\section{4 | FORMULATION WITH DIFFERENT REGIONS PROBLEM}

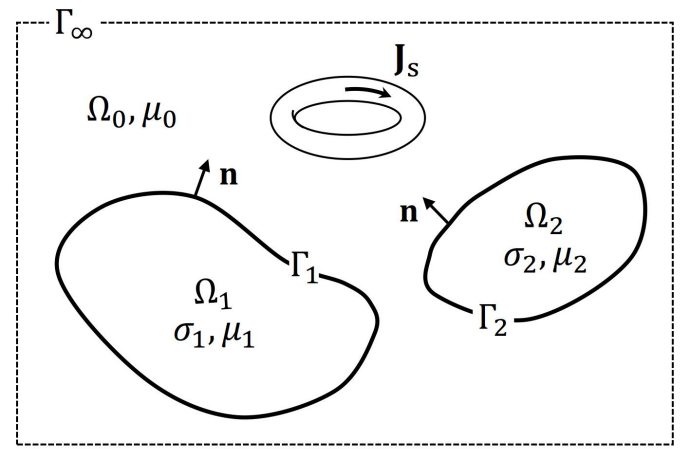

FIGURE 2 Problem with conductive electrical regions.

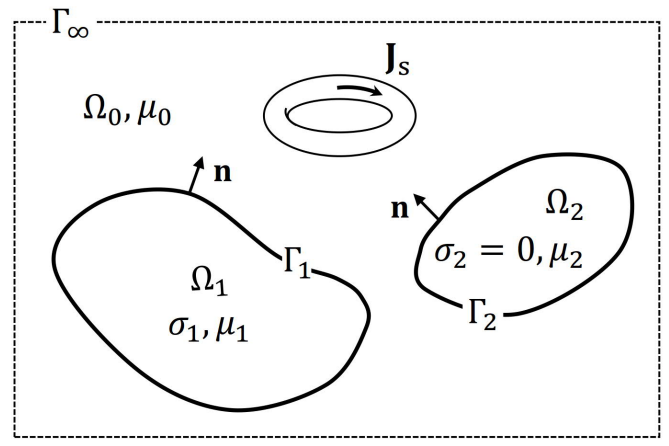

FIGURE 3 Problem with electrical and magnetic regions.

The $\mathbf{A}^{*}-\varphi$ BEM formulation can be applied to problems with different material regions. Let us consider quite different problems composed of two regions. In the case as in FIGURE 2 where all materials are conductive, we have $\left(\mathbf{n} \cdot \mathbf{A}^{*}\right)=0$ on its boundary. Thus, the integral equations for $\mathbf{A}^{*}$ on $\Gamma=\Gamma_{1} \cup \Gamma_{2}$ may be written in the form of 11 . We get the following system of equations:

$$
\left\{\begin{array}{l}
-\oint_{\Gamma} \mu\left(\mathbf{n} \times \mathbf{H}_{\mathrm{s}}\right) \mathrm{Gd} \Gamma=\mathrm{h} \mathbf{A}^{*}+\oint_{\Gamma}\left(\mathbf{n} \times \mathbf{A}^{*}\right) \times \nabla \mathrm{Gd} \Gamma-\mu \underset{\Gamma}{\oint_{\Gamma}}(\mathbf{n} \times \nabla \varphi) \mathrm{Gd} \Gamma \\
-\oint_{\Gamma}\left(\mathbf{n} \cdot \mathbf{H}_{\mathrm{s}}\right) \mathrm{G}_{0} \mathrm{~d} \Gamma=\mathrm{h}_{0} \varphi-\oint_{\Gamma}\left(\varphi\left(\mathbf{n} \cdot \nabla \mathrm{G}_{0}\right)+\frac{1}{\mu_{0}} \mathbf{n} \cdot\left(\nabla \times \mathbf{A}^{*}\right) \mathrm{G}_{0}\right) \mathrm{d} \Gamma
\end{array}\right.
$$

where the permeability $\mu$ and complex kernel G have values that depend on the characteristics of a domain of integration to be $\Gamma_{1}$ or $\Gamma_{2}$. The expression of $\mathrm{G}$ is 10 with the appropriate coefficient $\mathrm{k}$.

In the case of a problem as in FIGURE 3 a non-vanishing term $\left(\mathbf{n} \cdot \mathbf{A}^{*}\right)$ appears in the surface integral equation for $\mathbf{A}^{*}$ on $\Gamma_{2}$. In such a situation, we propose an extension version for the $\mathbf{A}^{*}-\varphi$ formulation. The surface integral equation in $\mathbf{H}^{11112}$ for $\Omega_{2}$ will be used instead of the equation in $\mathbf{A}^{*}$ :

$$
h_{2} \mathbf{H}=-\oint_{\Gamma_{2}}(\mathbf{n} \cdot \mathbf{H}) \nabla G_{0} d \Gamma-\oint_{\Gamma_{2}}(\mathbf{n} \times \mathbf{H}) \times \nabla G_{0} d \Gamma
$$

Using the continuity of the magnetic field across the boundary $\Gamma_{2}$, we obtain:

$$
-\mathbf{n} \cdot \oint_{\Gamma_{2}}\left(\mathbf{n} \times \mathbf{H}_{s}\right) \times \nabla G_{0} d \Gamma=h_{2}(\mathbf{n} \cdot \mathbf{H})+\mathbf{n} \cdot \oint_{\Gamma_{2}}(\mathbf{n} \cdot \mathbf{H}) \nabla G_{0} d \Gamma-\mathbf{n} \cdot \oint_{\Gamma_{2}}(\mathbf{n} \times \nabla \varphi) \times \nabla G_{0} d \Gamma
$$

The integral equation for magnetic region $\Omega_{2}$ is composed by two scalar variables (n.H) and $\varphi$. Combining it with the equation of $\mathbf{A}^{*}$ for region $\Omega_{1}$ and $\varphi$ for the air region, we obtain a new system of equations:

$$
\left\{\begin{array}{l}
-\oint_{\Gamma_{1}} \mu_{1}\left(\mathbf{n} \times \mathbf{H}_{\mathrm{s}}\right) \mathrm{Gd} \Gamma=\mathrm{h}_{1} \mathbf{A}^{*}+\oint_{\Gamma_{1}}\left(\mathbf{n} \times \mathbf{A}^{*}\right) \times \nabla \mathrm{Gd} \Gamma-\mu_{1} \oint_{\Gamma_{1}}(\mathbf{n} \times \nabla \varphi) \mathrm{Gd} \Gamma \\
-\oint_{\Gamma}\left(\mathbf{n} \cdot \mathbf{H}_{\mathrm{s}}\right) \mathrm{G}_{0} \mathrm{~d} \Gamma=\mathrm{h}_{0} \varphi-\oint_{\Gamma} \varphi\left(\mathbf{n} \cdot \nabla \mathrm{G}_{0}\right) \mathrm{d} \Gamma-\oint_{\Gamma_{1}} \frac{1}{\mu_{0}} \mathbf{n} \cdot\left(\nabla \times \mathbf{A}^{*}\right) \mathrm{G}_{0} \mathrm{~d} \Gamma-\oint_{\Gamma_{2}} \frac{\mu_{2}}{\mu_{0}}(\mathbf{n} \cdot \mathbf{H}) \mathrm{G}_{0} \mathrm{~d} \Gamma \\
-\underset{\Gamma_{2}}{\mathbf{n}} \cdot \oint_{\Gamma_{2}}\left(\mathbf{n} \times \mathbf{H}_{\mathrm{s}}\right) \times \nabla \mathrm{G}_{0} \mathrm{~d} \Gamma=\mathrm{h}_{2}(\mathbf{n} \cdot \mathbf{H})+\mathbf{n} \cdot \oint_{\Gamma_{2}}(\mathbf{n}) \nabla \mathrm{G}_{0} \mathrm{~d} \Gamma-\mathbf{n} \cdot \underset{\mathbf{n}}{\oint}(\mathbf{n}) \times \nabla \mathrm{G}_{0} \mathrm{~d} \Gamma
\end{array}\right.
$$

\section{5 | NUMERICAL IMPLEMENTATION}

The surface $\Gamma$ is discretized by first order plane elements. Edge shape functions and a nodal shape functions are used to interpolate the $\mathbf{A}^{*}$ and $\varphi$ respectively.

$$
\left\{\begin{array}{l}
\mathbf{A}^{*}=\sum_{\mathrm{i}} \mathbf{w}_{\mathrm{i}} A_{\mathrm{i}}^{*} \\
\varphi=\sum_{\mathrm{i}} \alpha_{\mathrm{i}} \varphi_{\mathrm{i}}
\end{array}\right.
$$


The Galerkin's approach is used with the edge shape functions $\mathbf{w}_{\mathrm{i}}$ and the nodal shape functions $\alpha_{\mathrm{i}}$ as projection functions for the equation of $\mathbf{A}^{*}$ and $\varphi$ respectively. Coefficients $h_{0}$ and $h_{1}$ are both set to 0.5 . We finally obtain a linear matrix system which has to be solved:

$$
\left[\begin{array}{ll}
\mathbf{M}^{11} & \mathbf{M}^{12} \\
\mathbf{M}^{21} & \mathbf{M}^{22}
\end{array}\right]\left\{\begin{array}{c}
\mathrm{A}^{*} \\
\varphi
\end{array}\right\}=\left\{\begin{array}{l}
\mathbf{N}^{1} \\
\mathbf{N}^{2}
\end{array}\right\}
$$

$$
\begin{array}{ll}
\mathbf{M}_{\mathrm{ij}}^{11}=0.5 \oint_{\Gamma} \mathbf{w}_{\mathrm{i}} \mathbf{w}_{\mathrm{j}} \mathrm{d} \Gamma+\oint_{\Gamma} \mathbf{w}_{\mathrm{i}} \oint_{\Gamma}\left(\mathbf{n} \times \mathbf{w}_{\mathrm{j}}\right) \times \nabla \mathrm{Gd} \Gamma \mathrm{d} \Gamma & \mathbf{M}_{\mathrm{ij}}^{22}=0.5 \oint_{\Gamma} \alpha_{\mathrm{i}} \alpha_{\mathrm{j}} \mathrm{d} \Gamma-\oint_{\Gamma} \alpha_{\mathrm{i}} \oint_{\Gamma}\left(\mathbf{n} \cdot \nabla \mathrm{G}_{0}\right) \alpha_{\mathrm{j}} \mathrm{d} \Gamma \mathrm{d} \Gamma \\
\mathbf{M}_{\mathrm{ij}}^{12}=-\mu \oint_{\Gamma} \mathbf{w}_{\mathrm{i}} \oint_{\Gamma}\left(\mathbf{n} \times \nabla \alpha_{\mathrm{j}}\right) \mathrm{Gd} \Gamma \mathrm{d} \Gamma & \mathbf{N}_{\mathrm{i}}^{1}=-\mu \oint_{\Gamma} \mathbf{w}_{\mathrm{i}} \oint_{\Gamma}\left(\mathbf{n} \times \mathbf{H}_{\mathrm{s}}\right) \mathrm{Gd} \Gamma \mathrm{d} \Gamma \\
\mathbf{M}_{\mathrm{ij}}^{21}=-\frac{1}{\mu_{0}} \oint_{\Gamma} \alpha_{\mathrm{i}} \oint_{\Gamma} \mathbf{n} \cdot\left(\nabla \times \mathbf{w}_{\mathrm{j}}\right) \mathrm{G}_{0} \mathrm{~d} \Gamma \mathrm{d} \Gamma & \mathbf{N}_{\mathrm{i}}^{2}=-\oint_{\Gamma} \alpha_{\mathrm{i}} \oint_{\Gamma}\left(\mathbf{n} \cdot \mathbf{H}_{\mathrm{s}}\right) \mathrm{G}_{0} \mathrm{~d} \Gamma \mathrm{d} \Gamma
\end{array}
$$

For the system [23, in addition to the approximations of $\mathbf{A}^{*}$ and $\varphi$ in [24], the new scalar variable (n.H) has to be added which is approximated by a nodal shape functions.

$$
\mathbf{n} \cdot \mathbf{H}=\sum_{i} \alpha_{i} h_{i}
$$

The matrix obtained by applying the Galerkin method is as follows:

$$
\left[\begin{array}{ccc}
\mathbf{M}^{11} & \mathbf{M}^{12} & 0 \\
\mathbf{M}^{21} & \mathbf{M}^{22} & \mathbf{M}^{23} \\
0 & \mathbf{M}^{32} & \mathbf{M}^{33}
\end{array}\right]\left\{\begin{array}{c}
A^{*} \\
\varphi \\
\mathbf{h}
\end{array}\right\}=\left\{\begin{array}{l}
\mathbf{N}^{1} \\
\mathbf{N}^{2} \\
\mathbf{N}^{3}
\end{array}\right\}
$$

$$
\begin{array}{lll}
\mathbf{M}_{\mathrm{ij}}^{11}=0.5 \oint_{\Gamma_{1}} \mathbf{w}_{\mathrm{i}} \mathbf{w}_{\mathrm{j}} \mathrm{d} \Gamma+\oint_{\Gamma_{1}} \mathbf{w}_{\mathrm{i}} \oint_{\Gamma_{1}}\left(\mathbf{n} \times \mathbf{w}_{\mathrm{j}}\right) \times \nabla \mathrm{Gd} \Gamma \mathrm{d} \Gamma & \mathbf{M}_{\mathrm{ij}}^{32}=0.5 \oint_{\Gamma_{2}} \alpha_{\mathrm{i}} \alpha_{\mathrm{j}} \mathrm{d} \Gamma-\oint_{\Gamma_{2}} \mathbf{n} \cdot \alpha_{\mathrm{i}} \oint_{\Gamma_{2}}\left(\mathbf{n} \cdot \nabla \mathrm{G}_{0}\right) \alpha_{\mathrm{j}} \mathrm{d} \Gamma \mathrm{d} \Gamma \\
\mathbf{M}_{\mathrm{ij}}^{12}=-\mu_{1} \oint_{\Gamma_{1}} \mathbf{w}_{\mathrm{i}} \oint_{\Gamma_{1}}\left(\mathbf{n} \times \nabla \alpha_{\mathrm{j}}\right) \mathrm{Gd} \Gamma \mathrm{d} \Gamma & \mathbf{M}_{\mathrm{ij}}^{33}=-\oint_{\Gamma_{2}} \mathbf{n} \cdot \alpha_{\mathrm{i}} \oint_{\Gamma_{2}}\left(\mathbf{n} \times \nabla \alpha_{\mathrm{j}}\right) \times \nabla \mathrm{G}_{0} \mathrm{~d} \Gamma \mathrm{d} \Gamma \\
\mathbf{M}_{\mathrm{ij}}^{21}=-\frac{1}{\mu_{0}} \oint_{\Gamma} \alpha_{\mathrm{i}} \oint_{\Gamma_{1}} \mathbf{n} \cdot\left(\nabla \times \mathbf{w}_{\mathrm{j}}\right) \mathrm{G}_{0} \mathrm{~d} \Gamma \mathrm{d} \Gamma & \mathbf{N}_{\mathrm{i}}^{1}=-\mu_{1} \oint_{\Gamma_{1}} \mathbf{w}_{\mathrm{i}} \oint_{\Gamma_{1}}\left(\mathbf{n} \times \mathbf{H}_{\mathrm{s}}\right) \mathrm{Gd} \Gamma \mathrm{d} \Gamma \\
\mathbf{M}_{\mathrm{ij}}^{22}=0.5 \oint_{\Gamma} \alpha_{\mathrm{i}} \alpha_{\mathrm{j}} \mathrm{d} \Gamma-\oint_{\Gamma} \alpha_{\mathrm{i}} \oint_{\Gamma}\left(\mathbf{n} \cdot \nabla \mathrm{G}_{0}\right) \alpha_{\mathrm{j}} \mathrm{d} \Gamma \mathrm{d} \Gamma & \mathbf{N}_{\mathrm{i}}^{2}=-\oint_{\Gamma} \alpha_{\mathrm{i}} \oint_{\Gamma}\left(\mathbf{n} \cdot \mathbf{H}_{\mathbf{s}}\right) \mathrm{G}_{0} \mathrm{~d} \Gamma \mathrm{d} \Gamma \\
\mathbf{M}_{\mathrm{ij}}^{23}=-\frac{\mu_{2}}{\mu_{0}} \oint_{\Gamma} \alpha_{\mathrm{i}} \oint_{\Gamma_{2}} \alpha_{\mathrm{j}} \mathrm{G}_{0} \mathrm{~d} \Gamma \mathrm{d} \Gamma & \mathbf{N}_{\mathrm{i}}^{3}=-\oint_{\Gamma_{2}} \mathbf{n} \cdot \alpha_{\mathrm{i}} \oint_{\Gamma_{2}}\left(\mathbf{n} \times \mathbf{H}_{\mathbf{s}}\right) \times \nabla \mathrm{G}_{0} \mathrm{~d} \Gamma \mathrm{d} \Gamma
\end{array}
$$

In order to accurately compute the integrals dealing with singular kernel $\mathrm{G}_{0}$ and its gradients, we can use the analytical expressions proposed by Graglia in $\sqrt{13}$ for a mesh composed of triangular elements. To perform the calculations related to $\mathrm{G}$, the kernel is split in two parts such as: $\mathrm{G}=$ $\mathrm{G}_{0}+\mathrm{G}_{1}$.

$$
G_{1}=\frac{e^{-(1+j) k r}-1}{4 \pi r}
$$

$\mathrm{G}_{1}$ and its gradient tend toward finite limits when $\mathrm{r}$ approaches zero as follows:

$$
\lim _{r \rightarrow 0}\left(G_{1}\right)=-\frac{\sqrt{\pi f \mu_{1} \sigma_{1}}}{4 \pi}-j \frac{\sqrt{\pi f \mu_{1} \sigma_{1}}}{4 \pi} ; \lim _{r \rightarrow 0}\left(\nabla G_{1}\right)=j \frac{f \mu_{1} \sigma_{1}}{4}
$$

Thus, the integrals related to $G_{1}$ can be simply computed by shifting Gauss point locations for the sources and the targets. Thus, the problem of singular integrals computation for $\mathrm{G}$ has been transferred to $\mathrm{G}_{0}$ which has an analytical expression.

\section{I NUMERICAL EXAMPLE}

\section{1 | TEST MODEL 1}

Let us consider a conductive-magnetic sphere of $10 \mathrm{~mm}$ radius excited by a uniform magnetic field $\mathbf{B}_{\mathrm{s}}=(0,0,1) \mathrm{T}$. The conductivity and permeability of the sphere are $\sigma=5.5 \mathrm{E} 7 \mathrm{~S} / \mathrm{m}$ and $\mu=10$ respectively. In this test, we focus on the calculation of Joule losses. With the proposed formulation, the losses are calculated by the expression 19 and will be compared to the losses obtained by other methods such as FEM, BEM-FEM 14 or SIBC (Surface Impedance Boudary Condition) 17! A volume mesh and a surface mesh with the same discretion of the surface will be used. A surface mesh with 2058 triangle elements is used for BEM and SIBC formulations while a volume mesh with 21231 tetrahedron elements is used for the others methods involving FEM. The size of each mesh elements is about $1.25 \mathrm{~mm}$. The comparison in terms of Joule losses is shown in TABLE 1 with the analytical solution proposed by Morisue ${ }^{2}$ considered as the reference value. 


\begin{tabular}{lccccc}
\hline \multirow{2}{*}{ Frequency $(\mathrm{Hz})$} & Skin depth $(\mathrm{mm})$ & \multicolumn{5}{c}{ Error (\%) } \\
\cline { 3 - 6 } & & $\mathbf{A}^{*}-\varphi$ BEM & BEM-FEM & SIBC & FEM \\
\hline 10 & 6.79 & 0.88 & 1.06 & 86.17 & 0.04 \\
100 & 2.15 & 0.33 & 0.77 & 0.37 & 1.32 \\
1000 & 0.68 & 0.18 & 13.37 & 2.64 & 12.6 \\
10000 & 0.21 & 0.51 & 24.83 & 1.54 & 46.94 \\
100000 & 0.07 & 0.40 & 75.96 & 0.75 & 82.46 \\
\hline
\end{tabular}

TABLE 1 Relative errors of Joule losses for different methods.

The accuracy of methods FEM and SIBC is strongly influenced by the volume mesh of the conductive region. The increase of the frequency making the skin depth decrease, the volume mesh would need to be refined in the context of FEM. The mesh being fixed, the accuracy becomes less good when the frequency increases with the BEM-FEM approach. The SIBC is well known to be relevant at high frequency because the air-conductor interface is approached by a semi-infinite plane. This is why its accuracy increases with the increase of the frequency. The BEM results (all relative errors being less than $1 \%$ ) shows that the accuracy of the method is very good on a large frequency spectrum compared to others approach. The low sensitivity of BEM to the skin depth is certainly one of its main advantage.

\section{2 | TEST MODEL 2}
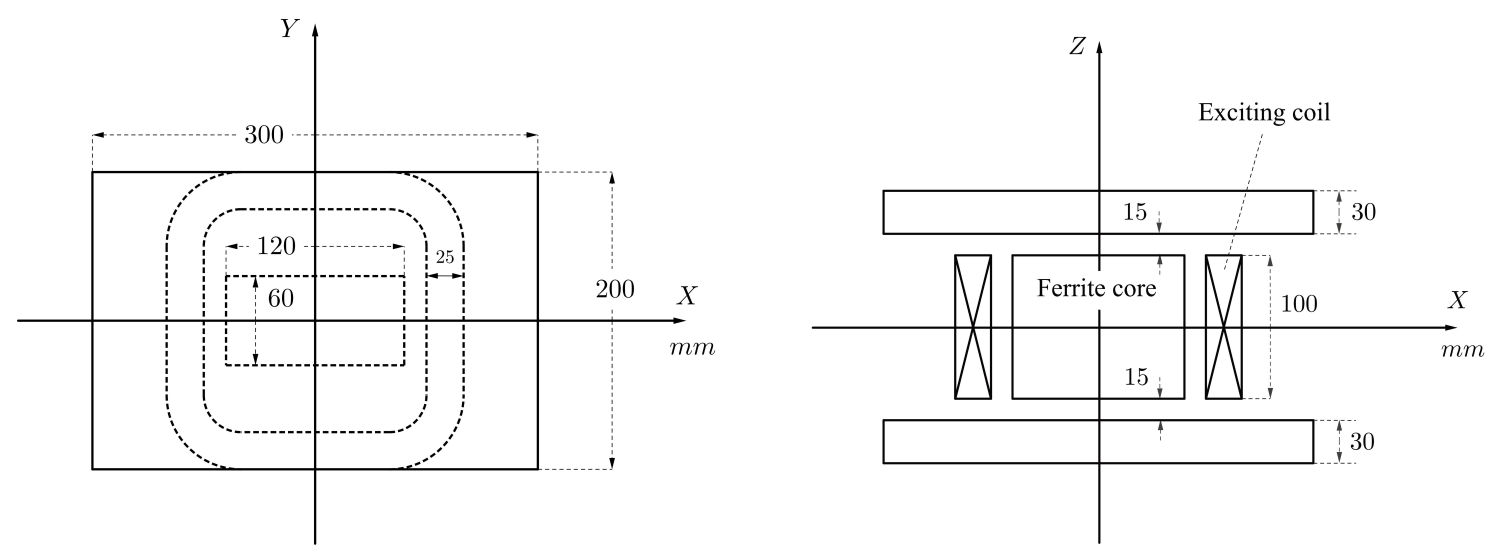

FIGURE 4 The model proposed by IEEJ.

In order to validate our formulation for the multi-region problem [23, the problem proposed by IEE of Japan ${ }^{14}$ is considered. The geometry of the problem is described in FIGURE 4 The conductivity of the two aluminum plates is $3.215 \mathrm{E} 7(\mathrm{~S} / \mathrm{m})$. The ferrite magnetic region has a relative linear permeability of 3000 . The excitation coil is fed by an alternating current $1000 \mathrm{~A}, 50 \mathrm{~Hz}$. A comparisons of magnetic field computed on a line located in the air region by BEM and BEM-FEM method is shown in FIGURE 5 For BEM formulation, 2800 quadrangle ared used in the surface meshes while BEM-FEM is based on a volume mesh for the material of 27860 hexahedrons. For the Joule losses, one more simulation was performed by FEM with an adapted mesh. The calculated results are shown in TABLE[2 In terms of memory requirements, the space required for FE method is about 5 times larger than that required for the proposed formulation. Theses results demonstrates the efficiency of the BEM in the context of more complicated geometry. 

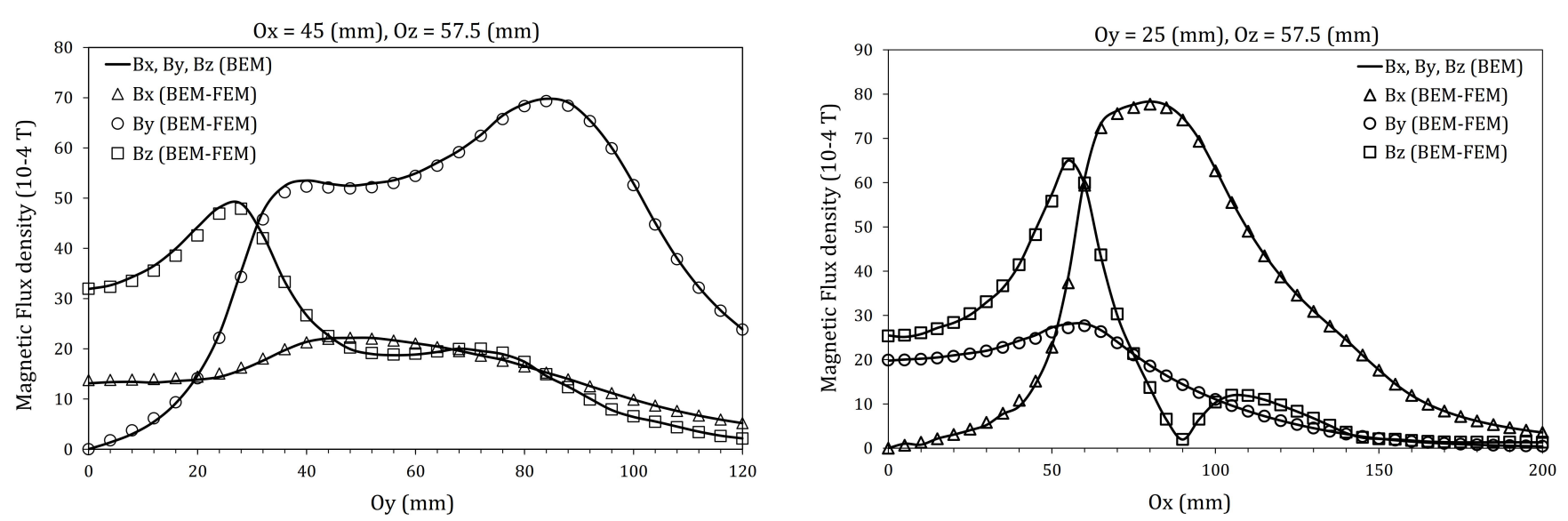

FIGURE 5 The magnetic flux density.

\begin{tabular}{lccc}
\hline & A $^{*}-\varphi$ BEM & BEM-FEM & A $-\phi$ FEM \\
\hline Joule losses (W) & 2.794 & 2.792 & 2.784 \\
\hline Difference (\%) & - & 0.071 & 0.358 \\
\hline
\end{tabular}

TABLE 2 The relative error of Joule losses.

\section{7 | CONCLUSIONS}

In this paper, we have presented a new BEM formulation based on the modified magnetic vector potential and the reduced magnetic scalar potential to calculate the eddy current. Since this formulation contains only one vector quantity and a single scalar quantity, the size of the matrix to be solved is not large. In addition, the accuracy of the method is less sensitive to the skin depth of the problem than with others methods where the mesh have to be adapted. Thus, in the case of small skin depth, the method does not lead to an explosion of memory and computing time caused by a very large number of mesh elements. The second test showed that the formulation can be also applied to problems with multi-regions and more complex geometry. However, the proposed formulation being based on reduced scalar magnetic potential in air, it is not appropriate to model problems containing non-simply connected conductive domains.

\section{References}

1. Unz H. Scalar-vector analog of Green's theorem. IRE Transactions on Antennas and Propagation. 1958;6(3):300-300. doi:10.1109/TAP.1958.1144586

2. Morisue T, Fukumi M. 3-D eddy current calculations using the magnetic vector potential. IEEE Transactions on Magnetics. 1988;24(1):106-109. doi:10.1109/20.43867

3. Rucker WM, Richter KR. A BEM code for 3-D eddy current calculations. IEEE Transactions on Magnetics. 1990;26(2):462-465. doi:10.1109/20.106353

4. Tsuboi H, Tanaka M. Three-dimensional eddy current analysis by the boundary element method using vector potential. IEEE Transactions on Magnetics. 1990;26(2):454-457. doi:10.1109/20.106351

5. Misaki T, Tsuboi H. Computaton of 3-dimensional Eddy current problems by using boundary element method. IEEE Transactions on Magnetics. 1985;21(6):2227-2230. doi:10.1109/TMAG.1985.1064200

6. Mayergoyz I. Boundary integral equations of minimum order for the calculation of three-dimensional eddy current problems. IEEE Transactions on Magnetics. 1982;18(2):536-539. doi:10.1109/TMAG.1982.1061855 
7. Kalaichelvan S, Lavers JD. On the implementation of a boundary element method for 3-D multiply connected EM field problems. IEEE Transactions on Magnetics. 1988;24(1):569-572. doi:10.1109/20.43975

8. Harrington RF. Formulation of Boundary Integral Equations by the Equivalent Source Method. In: Brebbia CA, Ingber MS, eds. Boundary Element Technology VII. Dordrecht: Springer Netherlands; 1992:293-303

9. Schmidlin G, Fischer U, Andjelic Z, Schwab C. Preconditioning of the second kind boundary integral equations for 3D eddy current problems. International Journal for Numerical Methods in Engineering. 2001;51(9):1009-1031. doi:10.1002/nme.187

10. Hiptmair R. Boundary Element Methods for Eddy Current Computation. In: Schanz M, Steinbach O, eds. Boundary Element Analysis. Vol 29. Berlin, Heidelberg: Springer Berlin Heidelberg; 2007:213-248. doi:10.1007/978-3-540-47533-0-9

11. Ishibashi K. A least residual approach for 3-D eddy current analysis by BEM. IEEE Transactions on Magnetics. 1993;29(2):1512-1515. doi:10.1109/20.250690

12. Zheng D. Three-dimensional eddy current analysis by the boundary element method. IEEE Transactions on Magnetics. $1997 ; 33(2): 1354-1357$. doi:10.1109/20.582507

13. Graglia RD. On the numerical integration of the linear shape functions times the 3-D Green's function or its gradient on a plane triangle. IEEE Transactions on Antennas and Propagation. 1993;41(10):1448-1455. doi:10.1109/8.247786

14. S. Wakao and T. Onuki, "Electromagnetic field computations by the hybrid FE-BE method using edge elements," in IEEE Transactions on Magnetics, vol. 29, no. 2, pp. 1487-1490, March 1993. doi: 10.1109/20.250684

15. T. Onuki and S. Wakao, "Novel boundary element analysis for 3-D eddy current problems," in IEEE Transactions on Magnetics, vol. 29, no. 2, pp. 1520-1523, March 1993. doi: 10.1109/20.250692

16. Stratton JA, Chu LJ. Diffraction Theory of Electromagnetic Waves.; 1939.

17. Z. De Greve, J. Siau, G. Meunier, J. Guichon and O. Chadebec, "A Mixed Surface Volume Integral Formulation for the Modeling of High-Frequency Coreless Inductors," in IEEE Transactions on Magnetics, vol. 52, no. 3, pp. 1-4, March 2016, Art no. 7208904. doi: 10.1109/TMAG.2015.2497004 